\title{
The replication origin of a repABC plasmid
}

\author{
Ramón Cervantes-Rivera, Francisco Pedraza-López, Gabriela Pérez-Segura and Miguel A Cevallos*
}

\begin{abstract}
Background: repABC operons are present on large, low copy-number plasmids and on some secondary chromosomes in at least $19 \alpha$-proteobacterial genera, and are responsible for the replication and segregation properties of these replicons. These operons consist, with some variations, of three genes: repA, repB, and repC. RepA and RepB are involved in plasmid partitioning and in the negative regulation of their own transcription, and RepC is the limiting factor for replication. An antisense RNA encoded between the repB-repC genes modulates repC expression.

Results: To identify the minimal region of the Rhizobium etli p42d plasmid that is capable of autonomous replication, we amplified different regions of the repABC operon using PCR and cloned the regions into a suicide vector. The resulting vectors were then introduced into $R$. etli strains that did or did not contain $\mathrm{p} 42 \mathrm{~d}$. The minimal replicon consisted of a repC open reading frame under the control of a constitutive promoter with a ShineDalgarno sequence that we designed. A sequence analysis of repC revealed the presence of a large A+T-rich region but no iterons or DnaA boxes. Silent mutations that modified the A+T content of this region eliminated the replication capability of the plasmid. The minimal replicon could not be introduced into $R$. etli strain containing p42d, but similar constructs that carried repC from Sinorhizobium meliloti pSymA or the linear chromosome of Agrobacterium tumefaciens replicated in the presence or absence of $\mathrm{p} 42 \mathrm{~d}$, indicating that RepC is an incompatibility factor. A hybrid gene construct expressing a RepC protein with the first 362 amino acid residues from p42d RepC and the last 39 amino acid residues of RepC from SymA was able to replicate in the presence of p42d.

Conclusions: RepC is the only element encoded in the repABC operon of the R. etli p42d plasmid that is necessary and sufficient for plasmid replication and is probably the initiator protein. The oriV of this plasmid resides within the repC gene and is located close to or inside of a large A+T region. RepC can act as an incompatibility factor, and the last 39 amino acid residues of the carboxy-terminal region of this protein are involved in promoting this phenotype.
\end{abstract}

\section{Background}

Proteins that are involved in the initiation of DNA replication are essential to cells. These proteins recognize the origin of replication, destabilize double-stranded DNA, and recruit the replisome, which is the machinery directly involved in DNA replication [1].

Both the activity and concentration of the initiator proteins are highly regulated because the genetic material needs to be replicated only once per generation. A failure in this process could accelerate the production of new DNA molecules with a concomitant increase in the number of new origins of replication, which could be

\footnotetext{
* Correspondence: mac@ccg.unam.mx

Programa de Genómica Evolutiva, Centro de Ciencias Genómicas, Universidad Nacional Autónoma de México, Apartado Postal 565-A, Cuernavaca, Morelos, México
}

used in new rounds of replication and leading to cell death (i.e., "runaway replication") [2].

Initiator proteins control the replication rate using several mechanisms that limit either their own synthesis or their availability. The initiator proteins can directly auto-regulate the transcription of their own genes or trigger the production of negative regulators, antisenseRNAs or proteins, which are co-transcribed with the initiator genes. The activity of the initiator proteins can be controlled by covalent modifications or by titrating out their availability using DNA sites that resemble origins of replication. In addition, the DNA initiation rate can be controlled by blocking or hiding the origins of replication $[3,4]$.

The initiation of replication of the Escherichia coli chromosome and of some of its plasmids has been 
studied extensively. However, our knowledge of other bacterial replication systems is limited. Research on new replicons that are not found in E. coli or its close relatives would yield new insights into the regulation of initiation of replication in bacteria. The present work concerns rep $A B C$ replicons, which are present on large, low copy-number plasmids and on some secondary chromosomes in at least $19 \alpha$-proteobacterial genera. Some bacterial strains contain more than one $\operatorname{rep} A B C$ replicon, indicating that this plasmid family encompasses several incompatibility groups [5-7].

The basic replicon of $\operatorname{rep} A B C$ plasmids is compact because all of the elements required for replication and segregation are encoded in a single operon, the $\operatorname{rep} A B C$ operon $[8,9]$. However, this operon is controlled by a complex regulatory mechanism. The first two genes of the rep $A B C$ operon encode for proteins belonging to a type Ia segregation system [10]. RepA and RepB have been implicated in the negative transcriptional regulation of the rep $A B C$ operon $[9,11]$.

$\mathrm{RepC}$ is a limiting replication factor and thus has been suggested to be the initiator protein $[8,12,13]$. The members of the $\operatorname{rep} A B C$ family contain a centromericlike sequence (parS) in three possible locations: downstream of and close to the stop codon of repC [14,15], between $\operatorname{rep} A$ and $\operatorname{rep} B$, or upstream of $\operatorname{rep} A[16,17]$. A conserved sequence between the $\operatorname{rep} B$ and rep $C$ genes is present in all known rep $A B C$ replicons and contains an antisense RNA (ctRNA) gene, the product of which negatively modulates the expression of RepC [18-20]. Regulatory role of the ctRNA depends on its pairing with the $\operatorname{rep} A B C$ mRNA. In the absence of the ctRNA, the mRNA section corresponding to the repB-repC intergenic region folds into a large stem-loop structure so that the predicted repC Shine-Dalgarno (SD) sequence and the rep $C$ initiation codon remain singlestranded, allowing rep $C$ translation. In contrast, when the ctRNA hybridizes with the rep $A B C$ mRNA, the rep $C$ leader sequence forms an intrinsic terminator, blocking repC transcription [21].

Many aspects of the biology of these plasmids remain unknown, especially the details of the replication or segregation of these genetic elements. In this paper, we demonstrate the following: A) RepC is the only element encoded in the repABC operon of the Rhizobium etli $\mathrm{p} 42 \mathrm{~d}$ plasmid (formally pRetCFN42d) that is necessary and sufficient for plasmid replication. B) $\mathrm{RepC}$ is an incompatibility factor. C) The RepC carboxy-terminal region is involved in the incompatibility phenotype. D) The origin of replication of the rep $A B C$ plasmid resides in a large $\mathrm{A}+\mathrm{T}$-rich region located at the central section of the $\operatorname{rep} C$ gene.

\section{Methods}

Plasmids, bacterial strains and growth conditions

The bacterial strains and the plasmids used in this work are described in Table 1. E. coli strains were grown at $37^{\circ} \mathrm{C}$ in Luria-Bertani medium. Rhizobium strains were grown at $30^{\circ} \mathrm{C}$ in PY medium supplemented with $1 \mathrm{mM}$ $\mathrm{CaCl}_{2}$ [22]. Nalidixic acid $(20 \mu \mathrm{g} / \mathrm{ml})$ and chloramphenicol $(30 \mu \mathrm{g} / \mathrm{ml})$ were added when required. Growth kinetics were made in $500 \mathrm{ml}$ flasks containing, $50 \mathrm{ml}$ of PY medium without antibiotics. Incubation was performed at $30^{\circ} \mathrm{C}$ and $250 \mathrm{rpm}$.

\section{Bacterial mating}

pDOP derivatives were introduced into Rhizobium by conjugation using E. coli S17-1 as a donor strain [23]. The strains were grown in the proper antibiotic-free liquid medium to stationary phase, mixed in a donorrecipient ratio of $1: 2$ on antibiotic-free PY plates, and incubated at $30^{\circ} \mathrm{C}$ overnight. The cells were resuspended in PY medium, and serial dilutions were plated on the appropriate selective PY medium.

\section{Constructions}

The primers used in this work are presented in Table 2. The plasmid pDOP-H3 replicon was obtained by cloning a $5.6 \mathrm{~Kb}$ HindIII fragment containing the complete rep $A B C$ operon from $\mathrm{pH} 3$ into the HindIII site of pDOP [24]. The inserts of the plasmids used in this work, unless otherwise indicated, were PCR amplified from rep $A B C$ operon cloned in $\mathrm{pH} 3$ [24]. Inserts were introduced into $\mathrm{pDOP}$ in the sense orientation with respect to the promoter, using restriction sites that were included in the primer sequences.

\section{Constructs used to determine the minimal replicon}

Insert of plasmid pDOP- $\alpha \mathrm{C}$ was generated by amplifying the inc $\alpha-\operatorname{rep} C$ region with the primers ALFAU2 and Mal-C2Kpn. The repC (p42d) gene present in pDOP-C was amplified by PCR with the primers RBS$\mathrm{C}$ and Mal-C2. The repC gene of pSymA, present in construct pDOP-CsA, was obtained by PCR with the primers C-SymA and K-Syma-L and the genomic DNA of S. meliloti 2011 as the template. The repC gene of the linear chromosome of Agrobacterium tumefaciens C58 was obtained by PCR with the primers repCATBamU and repCATHinL and genomic DNA as the template.

\section{Constructs carrying repC deletions or repC fragments}

The insert of the plasmid pDOP-C/D1UM with a deletion in its 5 -end was obtained with the oligonucleotides repC-D1U and Mal-C2. The repC gene present in the plasmid $\mathrm{pDOP}-\mathrm{C} / \mathrm{RD} 1 \mathrm{~L}$ was amplified with the primers RBS-C and repC-D1L. Six plasmids carrying fragments of the repC gene were constructed: pDOP-C/F1 insert 
Table 1 Bacterial strains and plasmid used in this work

\begin{tabular}{|c|c|c|}
\hline Strain & Relevant characteristics & Reference \\
\hline $\begin{array}{l}\text { Rhizobium etli } \\
\text { CE3 }\end{array}$ & Streptomycin resistant derivative of CFN42 strain & {$[20]$} \\
\hline $\begin{array}{l}\text { R. etli } \\
\text { CFNX101 }\end{array}$ & recA:: $\Omega$-Spectinomycin derivative of CE3 & [46] \\
\hline $\begin{array}{l}\text { R. etli } \\
\text { CFNX107 }\end{array}$ & recA:: $\Omega$-Spectinomycin derivative of CE3, laking plasmid p42a and p42d. & [46] \\
\hline E. coli S17-1 & Plasmid donor in conjugations & [23] \\
\hline
\end{tabular}

pDOP A chloramphenicol resistant suicide vector derived from $\mathrm{pBC}$ SK(+), and containing oriT

pDOP-E' PDOP derivative with the intergenic region repB-repC, the complete repC gene under Placpromoter, and $500 \mathrm{pb}$

Reference downstream repC stop codon.

pDOP-H3 PDOP derivative carrying a $5.6 \mathrm{~Kb}$ Hindlll with repABC operon of $R$. etli plasmid p42d.

$\mathrm{pDOP}-\alpha \mathrm{C}$ pDOP derivative with the intergenic region repB-repC and the complete repC gene under Plac promoter.

PDOP-C pDOP carrying repC gen of plasmid p42d, with a SD sequence (AGGA) and under Plac promoter.

PDOP-C/ D1UM

PDOP-C/RD1L Similar to pDOP-C but with a repC gene carrying a deletion from codon 372 to codon 401

pDOP-F1 PDOP containing a repC fragment from codon 2 to codon 110, with a SD consensus sequence under Plac promoter.

pDOP-C/F1-F2 pDOP containing a repC fragment from codon 2 to codon 209, with a SD consensus sequence under Plac promoter.

pDOP-C/F1-F3 pDOP containing a repC fragment from codon 2 to codon 309, with a SD consensus sequence under Plac promoter.

PDOP-C/F4 pDOP containing a repC fragment from codon 310 to codon 403, with a SD consensus sequence under Plac promoter.

pDOP-C/F4-F3 pDOP containing a repC fragment from codon 210 to codon 403, with a SD consensus sequence under Plac promoter.

pDOP-C/F4-F2 pDOP containing a repC fragment from codon 111 to codon 403, with a SD consensus sequence under Plac promoter.

PDOP-C S/SD Similar to PDOP-C but without the SD sequence

PDOP-TtMC Similar to PDOP-C but with a mutant repC gene carrying

This work

[22]

This work

This work

This work

This work

This work

This work

This work

This work

This work

This work

This work

This work

This work

silent mutations to increase its CG content

pDOP-CBbglll Similar to pDOP-C but with repC gene, carrying a frameshift mutation at the Bg/ll restriction site

This work

pDOP-CSphl Similar to pDOP-C but with repC gene, carrying a frameshift mutation at the Sphl restriction site

This work

PDOP-CAtLC PDOP derivative carrying repC gen of the Agrobacterium

This work

tumefaciens C58 linear chromosome, with a SD sequence (AGGA) and under Plac promoter.

pDOP-CsA PDOP derivative carrying repC gen of the Sinorhizobium meliloti 1021 pSymA, with a SD sequence (AGGA) and under

This work Plac promoter.

pDOP/C420- pDOP with a hybrid repC gene, encoding the first 140 amino acid residues of the pSymA RepC protein and the rest of This work $1209 \quad$ p42d.

pDOP/C1-420 pDOP with a hybrid repC gene, encoding the first 140 amino acid residues of the p42d RepC protein and the rest of PSymA.

pDOP/C421- $\quad$ pDOP with a hybrid repC gene encoding, the first 140 amino acid residues of the pSymA RepC protein, the next 140

840 amino acid residues from the p42d RepC protein and the rest from the pSymA RepC protein.

pDOP/Cs421- pDOP witha hybrid repC gene, encoding the first 140 amino acid residues of the p42d RepC protein, the next $140 \quad$ This work $840 \quad$ amino acid residues from the pSymA RepC protein and the rest from the p42d RepC protein.

pDOP/C841- $\quad$ pDOP derivative with a hybrid repC gene, encoding the first 280 aminoacid residued of pSymA RepC and the rest of This work $1209 \quad$ p42d RepC protein.

pDOP/C1-990 pDOP derivative with a hybrid repC gene, encoding the first 330 amino acid residues of p42d RepC protein and the rest This work of pSymA RepC protein.

pDOP/C1-1086 pDOP derivative with a hybrid repC gene, encoding the first 362 amino acid residues of p42d RepC protein and the rest This work of pSymA RepC protein.

was obtained with primers repC-F1U and repC-F1L. The insert of plasmid pDOP-C/F1-F2 was obtained with primers repC-F1U and repC-F2L. Inserts of plasmids pDOP-C/F1-F3, pDOP-C/F4, pDOP-C/F4-F3, and pDOP-C/F4-F2 were obtained with the following primer pairs: repC-F1U and repC-F3L, repC-F4U and repC-
F4L, repC-F3U and repC-F4L, repC-F2U and repC-F4L, respectively.

\section{Construction of a repC mutant lacking its Shine-Dalgarno sequence}

The insert of the plasmid pDOP-Cs/SD was acquired by PCR with the primers repCd-sSDU and Mal-C2. 
Table 2 Oligonucleotides used in these work

\begin{tabular}{|c|c|}
\hline Name & Sequence \\
\hline ALFAU2 & 5'-AGGGTACCCCGCAAAAGAAAAGA \\
\hline Mal-C2Kpn & 5'-TCGGTACCTTACCCAGCCCTCAAACC \\
\hline RBS-C & 5'-GGATCCAAGGAAACAGCTATGCAGTCGGGGAATG \\
\hline repC-F1U & 5'-GCGGCCGCGGATCCAATGCAGTCGGGGAATGTA \\
\hline repC-F1L & 5'-ACTAGTCCCGGGAACCCCGACTCCACCAGA \\
\hline repC-F2U & 5'-GCGGCCGCGGATCCAATGGATCGTCCGTAAGGATAG \\
\hline repC-F2L & 5'-ACTAGTCCCGGGGCGCGGAATTCTGCTCGC \\
\hline repC-F3U & 5'-GCGGCCGCGGATCCAATGGTTCCGACCCTTGAAGGG \\
\hline repC-F3L & 5'-ACTAGTCCCGGGGCGCGGTGCATAGTCGCT \\
\hline repC-F4U & 5'-GCGGCCGCGGATCCAATGGGCGTGGGAAGCTGGCGA \\
\hline repC-F4L & 5'-ACTAGTCCCGGGTTACCCAGCCCTCAAACC \\
\hline repC-D1U & 5'-GGATCCAAGGAAACAGCTATGACGCTTGCGCTCGTGC \\
\hline repC-D1L & 5'-GCCAAGCTITTATATCATCGGGCCAAGC \\
\hline repC-D2U & 5'-GGATCCAAGGAAACAGCTATGGGAAAAGCTGCCGATA \\
\hline repC-D2L & 5'-GCCAAGCTITTATAGATCCCGCAGATAG \\
\hline repC-D3U & 5'-GGATCCAAGGAAACAGCTATGGAACTGCTCAAGA \\
\hline repC-D3L & 5'-GCCAAGCTTITATTCCAGGATGCACGCA \\
\hline repCd-sSDU & 5'-GAAAAGAGCTCCCTCAACGT \\
\hline Cd-StopC-U & 5'-GGATCCTAACAGTCGGGGAATG \\
\hline \multirow[t]{2}{*}{ Ttrack1-U } & 5'AGCCCGAGTCCGTGAACGAGTCCGAGCCGCGCTCCGAGAAG \\
\hline & GAGCAGCACATACAGAATTCAAAACCC \\
\hline \multirow[t]{2}{*}{ Ttrack1-L } & 5'-CTCGAGGTTCTCCAAGCGGTTCAGCACCTCCTCGCGGAG \\
\hline & CATCTCCATCTCGTTGAGCACGCTAGTGACCCCTTCAAG \\
\hline Ttrack2-U & 5'-ATCGAGCAGCACATCCAGAACTCCAAGCCCGAGTCCGTG \\
\hline \multirow[t]{2}{*}{ Ttrack2-L } & 5'-GGAGTTCTGGATGTGCTGCTCGATGTGGGCGGCGTTGGT \\
\hline & GCTGATCTTCTCGGCGTTCTCGAGGTTCTCCAAGCGGTT \\
\hline repCATBamU & 5'-GGATCCAAGGAAACAGCTATGGACAGCACATGTGTAACG \\
\hline repCATHindL & 5'-AAGCTTCTAACCCGCCATGCCCACCTC \\
\hline K-Syma-L & 5'-GGTACCTCACGACACCCCCCGCCC \\
\hline C-SymA & 5'-GGATCCAAGGAAACAGCTATGGAGATTGGAAGTGTGACG \\
\hline Mal-C2 & 5'-TCAAGCTITTACCCAGCCCTCAAACC \\
\hline$A L-2 U$ & 5'-ATCGGCACAGCGTTCGGCTITCGTCGCCCCTC CTGGCGCGATCGGAA \\
\hline $1 \mathrm{~L}-\mathrm{BU}$ & 5'-GAGAATGCTTTTGGCTTCGACCTGCTCGCACCAC TGCTGGCCCGCTCC \\
\hline $2 \mathrm{~L}-\mathrm{CU}$ & $\underline{5^{\prime}-G A G C A G G G G G C A A A G G C G A G C T T G G A A C C G G C C \text { AACAAGGCAAAAAGG }}$ \\
\hline$B L-3 U$ & 5'-GAGCGGTTAGACGGCCAAGCCATTAGCCTTCAGC CGAAGAATGAATCG \\
\hline AL-2UC & 5'-CGAAAAGCCGAACGCTGTGCCGAT \\
\hline $1 \mathrm{~L}-\mathrm{BUC}$ & 5'-CAGGTCGAAGCCAAAAGCATTCTC \\
\hline $2 \mathrm{~L}-\mathrm{CUC}$ & 5'-CAAGCTCGGCTITGCCCCCTGCTC \\
\hline BL-3Uc & 5'-AATGGCTTGGCCGTCTAACCGCTC \\
\hline Cd-1086L & 5'-GTTGATGAAGTTAGCCCTTTCCAG \\
\hline SaU-CdL & 5'-AACGCCAAGCATAGATCGTACCGT CCCCAGCATAGATCGAACCACCAC \\
\hline SaL-CdU & 5'-CTGTTGAGCTTCTATCCGGAGAAC GAGTTGCGTCAGGATGCACAATTG \\
\hline Cs-1087U & 5'-CTGGAAAGGGCTAACTTCATCAA CTCTGCTGGCGGCTATCTGCGCGAT \\
\hline
\end{tabular}

\section{Constructs carrying repC frame-shift mutations}

Plasmid pDOP-CBglII, was constructed digesting pDOP$\mathrm{C}$ with $B g l \mathrm{II}$ and filling in 5'-overhangs with T4 DNA polymerase (Fermentas). The blunted plasmid was ligated again with T4 ligase (Fermentas). Plasmid pDOP-CSphI was constructed in a similar way but digesting pDOP-C with SphI.
Construction of a repC gene carrying synonomous mutatios in the $A+T$ rich region

The pDOP-TtMC insert was obtained by an overlap extension PCR as described by Horton et al. (1989) [25]. The first PCR was performed using the primers Ttrack1-U and Mal-C2Kpn, and pH3 DNA as initial template. This product was purified and used as 
template for a second PCR with the oligonucleotides MalC2Kpn and Ttrack2-U; the amplification product was named T2-U. A third PCR amplification product obtained with the primers RBS-C and Ttrack1-L, and pH3 DNA as the template, was purified and used as a template in a new PCR reaction with the primers $\mathrm{RBS}-\mathrm{C}$ and Ttrack2- $\mathrm{L}$. The amplification product was named T2-L.

Finally, PCR products T2-U and T2-L were then mixed and used as the template for the last PCR. In this reaction, the primers $\mathrm{Mal}-\mathrm{C} 2 \mathrm{Kpn}$ and $\mathrm{RBS}-\mathrm{C}$ were used, and the final PCR product was cloned into pDOP.

\section{Construction of repC hybrid genes}

Overlap extension PCR was also employed to obtain repC hybrid genes. RepC gene amplification products from $\mathrm{pSymA}$ were obtained using pDOP-CsA as the template, and the $\operatorname{rep} \mathrm{C} 42 \mathrm{~d}$ products were obtained using pH3 as the template. Most of the hybrid genes described here required the overlap of two PCR products. The insert of plasmid pDOP/C420-1209 was obtained using the primers C-SymA and AL-2Uc for the first PCR product and AL-2U and Mal-C2 for the second product. The final PCR product was obtained with the external primers C-SymA and Mal-C2. The insert of plasmid pDOP/C1-420 was constructed with primers RBS-C and $1 \mathrm{~L}-\mathrm{B} 2 \mathrm{C}$ and the primers $1 \mathrm{~L}-\mathrm{B} 2$ and $\mathrm{K}-$ SymAL for the first and second PCR products, respectively. These products were combined using the primers RBS-C and K-SymAL. The pDOP/C841-1209 insert was constructed with the primers C-SymA and BL-3Uc for the first PCR product and BL-3U and Mal-C2 for the second. These products were joined in a third PCR with the primers C-SymA and Mal-C2. The hybrid gene in pDOP/C1-990 was acquired with the primers RBS-C and Sal-CdL for the first PCR product and Sal-CdU and Mal-C2 for the second. These PCR products were integrated in a third PCR with the primers RBS-C and MalC2. Similarly, the hybrid gene of pDOP/C1-990 was obtained with the primers RBS-C and Cd-1086 for the first amplification product. To obtain the second PCR product, the primers Cs-1087U and Mal-C2 were used, and both PCR products were fused with the primers RBS-C and Mal-C2. The inserts of two of the constructs, pDOP/C421-840 and pDOP/Cs421-840, required the fusion of three PCR products. The hybrid gene located in $\mathrm{pDOP} / \mathrm{C} 421-840$ required the primers C-SymA and AL-2Uc for the first PCR product, the primers AL-2U and AL-2Uc for the second PCR product, and the primers $2 \mathrm{~L}-\mathrm{CU}$ and $\mathrm{K}-\mathrm{SymA}$ for the third PCR product. The three PCR products were fused in the final PCR with the primers C-SymA and K-SymA. The hybrid gene present in $\mathrm{pDOP} / \mathrm{Cs} 421-840$ was obtained using the primers RBS-C and $1 \mathrm{~L}-\mathrm{B} 2 \mathrm{c}$ for the first PCR product, the primers $1 \mathrm{~L}-\mathrm{B} 2$ and $\mathrm{B} 2-3 \mathrm{Uc}$ for the second $\mathrm{PCR}$ product, and the primers $\mathrm{BL}-2 \mathrm{U}$ and $\mathrm{Mal}-\mathrm{C} 2$ for the third PCR product. These PCR products were linked using the primers RBS-C and Mal-C2 in the final PCR. DNA sequences of the inserts of all constructs were obtained to corroborate their correctness.

\section{Plasmid profiles}

The plasmid profiles of four transconjugants from each cross were visualized on agarose gels according to the protocol described by Hynes and McGregor [26].

\section{DNA isolation and manipulation}

Plasmid DNA was isolated using the High Pure Plasmid Isolation Kit (Roche) according to the manufacturer's instructions. Restriction and ligation reactions were performed under the conditions specified by the enzyme manufacturer (Fermentas). PCR was performed using Platinum High Fidelity Taq Platinum Polymerase or ThermalAce $^{\mathrm{TM}}$ DNA Polymerase (Invitrogen). PCR products were cloned using the TOPO TA Cloning Kit (Invitrogen).

\section{Plasmid incompatibility}

The incompatibility properties of the constructs were determined as described in Ramírez-Romero et al. [7].

\section{Plasmid replication in $R$. etli}

To determine the replication capabilities of the pDOP derivatives in $R$. etli, the plasmids were introduced into CFNX107 by conjugation. The plasmid profiles of at least four transconjugants from each cross were analyzed. A recombinant plasmid was considered capable of replicating in $R$. etli if the plasmid profiles of the transconjugants showed a new band of the expected size.

\section{Plasmid copy-number determination}

The plasmid copy numbers of the CFNX107 transconjugants containing pDOP derivatives were evaluated as follows: the total DNA of each transconjugant was isolated, digested with HindIII endonuclease, resolved in a $1 \%$ agarose gel and transferred to Hybond- + membranes (Amersham). The blot was then simultaneously hybridized with an $\Omega$ - spectinomycin cassette located within the $\operatorname{rec} A$ gene (chromosome-encoded) and with a fragment of pDOP; both probes were of the same size and GC content. The hybridization signals were quantified with a PhosphorImager SI (Molecular Dynamics). The plasmid copy-number was calculated from the ratio of the integrated hybridization signal of the recombinant plasmid and the integrated hybridization signal of the chromosome.

\section{Bioinformatics}

Alignments were performed with Clustal-W [27] at the WWW service of the European Bioinformatics Institute http://www2.ebi.ac.uk/clustalw. Protein secondary 
structure predictions were made with PSIPRED [28] at the WWW service of the Bioinformatics Group, UCL Department Of Computer Science http://bioinf.cs.ucl.ac. uk/psipred/. The DNA duplex helical stability profile was calculated using WEB-THERMODYN: sequence analysis software for profiling DNA helical stability http://www .gsa.buffalo.edu/dna/dk/WEBTHERMODYN/[29].

\section{Results}

\section{The oriV of $\mathrm{p} 42 \mathrm{~d}$ is located within the repC coding sequence}

The basic replicon of Rhizobium etli p42d, defined as the smallest DNA region that contains all of the elements required to replicate with the same stability and plasmid copy-number as the parental plasmid, consists of the complete repABC operon plus 500 bp downstream of the repC stop codon (inc-beta region, containing $p a r S$ ) and 86 bp upstream of the $\operatorname{rep} A$ initiation codon [8] (Figure 1). pDOP-E', a construct which carries the complete $r e p C$ gene, the intergenic sequence between repB and repC (inc-alpha), and the $500 \mathrm{bp}$ downstream of the repC stop codon under a constitutive promoter (Plac promoter), can replicate but does so with a slightly higher plasmid copy-number than the parental plasmid. However, derivatives that lack parts of the gene encoding the antisense RNA were unable to replicate [20].

To identify the minimal region of $\mathrm{p} 42 \mathrm{~d}$ that is capable of independent replication (putting aside the properties of the parental plasmid), we further explored the region between the repB stop codon and the 500 bp downstream of the repC stop codon. Three PCR products that possessed parts of this region were amplified and cloned into pDOP, a mobilizable suicide vector, under the control of the Plac promoter, which behaves as a constitutive promoter in Rhizobium. The first construct (pDOP- $\alpha \mathrm{C}$ ) contained the repB-repC intergenic region (inc-alpha) and the complete $\operatorname{rep} C$ gene. The second construct, pDOP-SDnC, contained the repC open reading frame (ORF), including its putative repC Shine-Dalgarno (SD) sequence (AGGUG). The third construct contained the repC ORF but with a SD sequence that was more similar to the Rhizobium etli SD consensus (AGGAA) positioned 6 bp prior to the $\operatorname{repC}$ initiation codon (pDOP-C). As a control, we introduced a HindIII fragment of $5.6 \mathrm{~Kb}$ that carried the entire $\operatorname{rep} A B C$ of $\mathrm{p} 42 \mathrm{~d}$ into pDOP conferring it the ability to replicate in Rhizobium (Figure 1) [24].

These constructs were introduced by mating into a recA Rhizobium etli CFN42 derivative lacking the $\mathrm{p} 42 \mathrm{~d}$ and $\mathrm{p} 42 \mathrm{a}$ plasmids (CFNX107) (Figure 1). Only constructs pDOP-H3, pDOP- $\alpha \mathrm{C}$ and pDOP-C were introduced with similar conjugation frequencies, from $1.6 \times 10^{-}$ ${ }^{3}$ to $6 \times 10^{4}$. However, CFNX107/pDOP-C transconjugants formed colonies after a longer time period (6-7 days), which was slower than the CFNX107/pDOP- $\alpha$ C and CFNX107/pDOP-H3 transconjugants and the receptor strain CFNX107 (3-4 days). Plasmid profile analyses of the transconjugants showed that the introduced plasmids replicated independently (Figure 2). The analyses also showed that $\mathrm{pDOP}-\mathrm{C}$ replicated with a higher plasmid copy-number than pDOP-H3 carrying the complete p42d repABC operon. This observation was corroborated by measuring the plasmid copy-number of the transconjugants: 6 copies of pDOP-C were present per chromosome instead of 1-2 copies of the control plasmid pDOP-H3 (Figure 3).

These results indicate that the minimal replicon of $\mathrm{p} 42 \mathrm{~d}$ consists of a repC gene under a constitutive promoter (Plac) and the SD sequence that we introduced and that the origin of replication resides within the repC-coding region. However, the growth rate of CFNX107 strain was negatively influenced by the introduction of pDOP-C (see Figure 4).

To prove that $\mathrm{RepC}$ is essential for replication, two repC deletions and two frame-shift mutants were constructed and cloned into pDOP under the control of the Plac promoter. Plasmid pDOP-C/D1UM contained a repC gene with a deletion of 14 codons (from codon 2 to 14 ), and plasmid pDOP-C/RD1L contained a repC gene with a deletion at its 3'end of 14 codons (from codon 388 to 401). The construct pDOP-CBglII possessed a repC gene with a frame-shift mutation at nucleotide 948, while plasmid pDOP-CSphI carried a frame-shift mutation at nucleotide 277. All of these constructs contained the same SD sequence as construct pDOP-C and were in the same relative orientation with respect to PLac in the vector. All plasmids were mated into the $R$. etli CFNX107 strain, but no transconjugants were obtained, indicating that the complete RepC product is crucial for replication.

To demonstrate that these observations were not specific to the p42d repC sequence, the repC genes of $S$. meliloti 1021 pSymA and the A. tumefaciens C58 linear chromosome were amplified by PCR and introduced into pDOP under Plac control and downstream of a SD sequence. The recombinant plasmids were conjugated into $R$. etli strain CFNX107, and the plasmid profiles of the transconjugants were analyzed. Both recombinant plasmids were capable of replication in Rhizobium, as was pDOP-C (Figure 2). These results clearly suggest that the presence of an origin of replication (oriV) within $r e p C$ is a general property of repABC operons.

\section{Analysis of the repC sequence: the role of the high $\mathrm{A}+\mathrm{T}$ content region}

To circumscribe the origin of replication (oriV) of the rep $A B C$ plasmids, we performed an in silico analysis to 


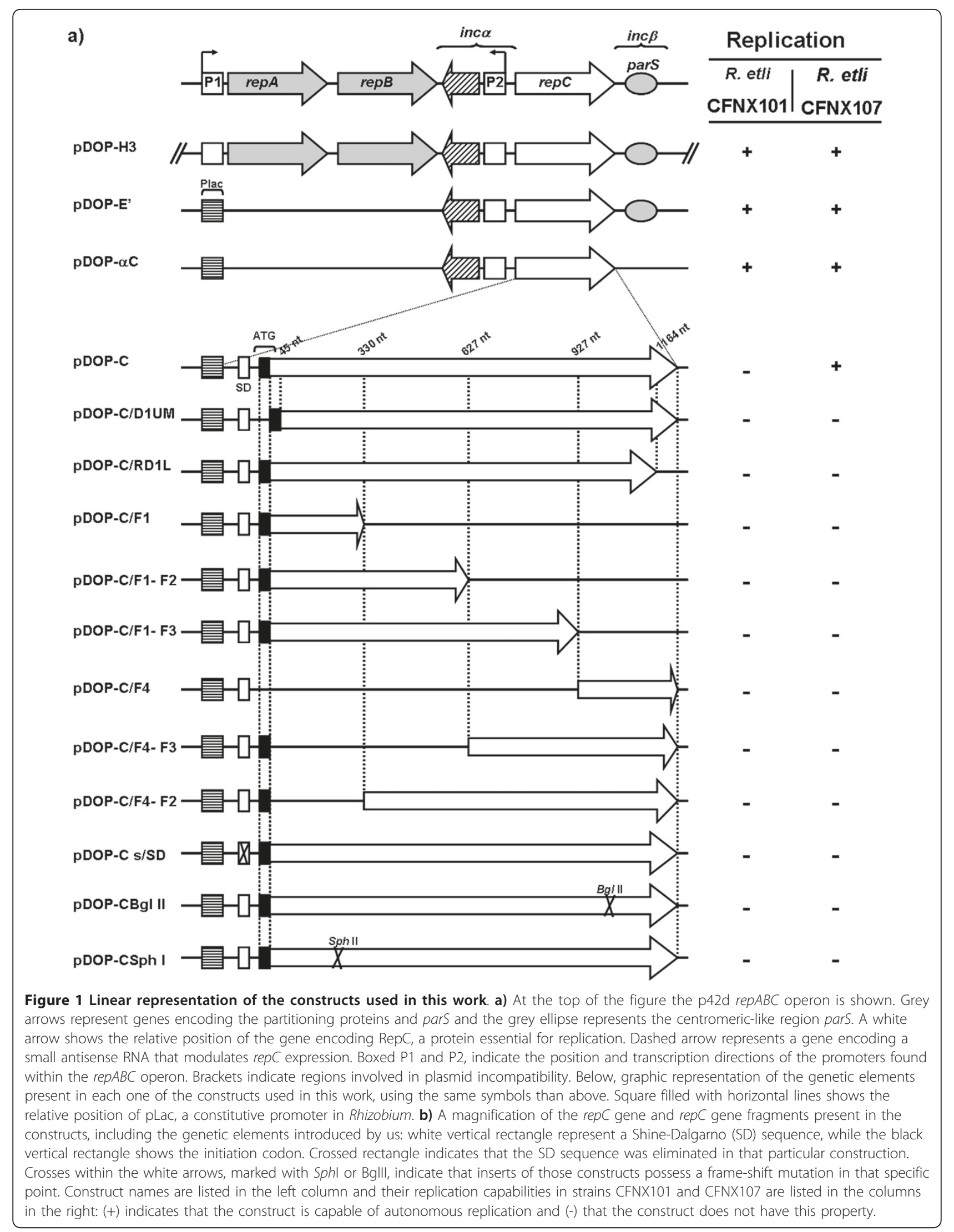




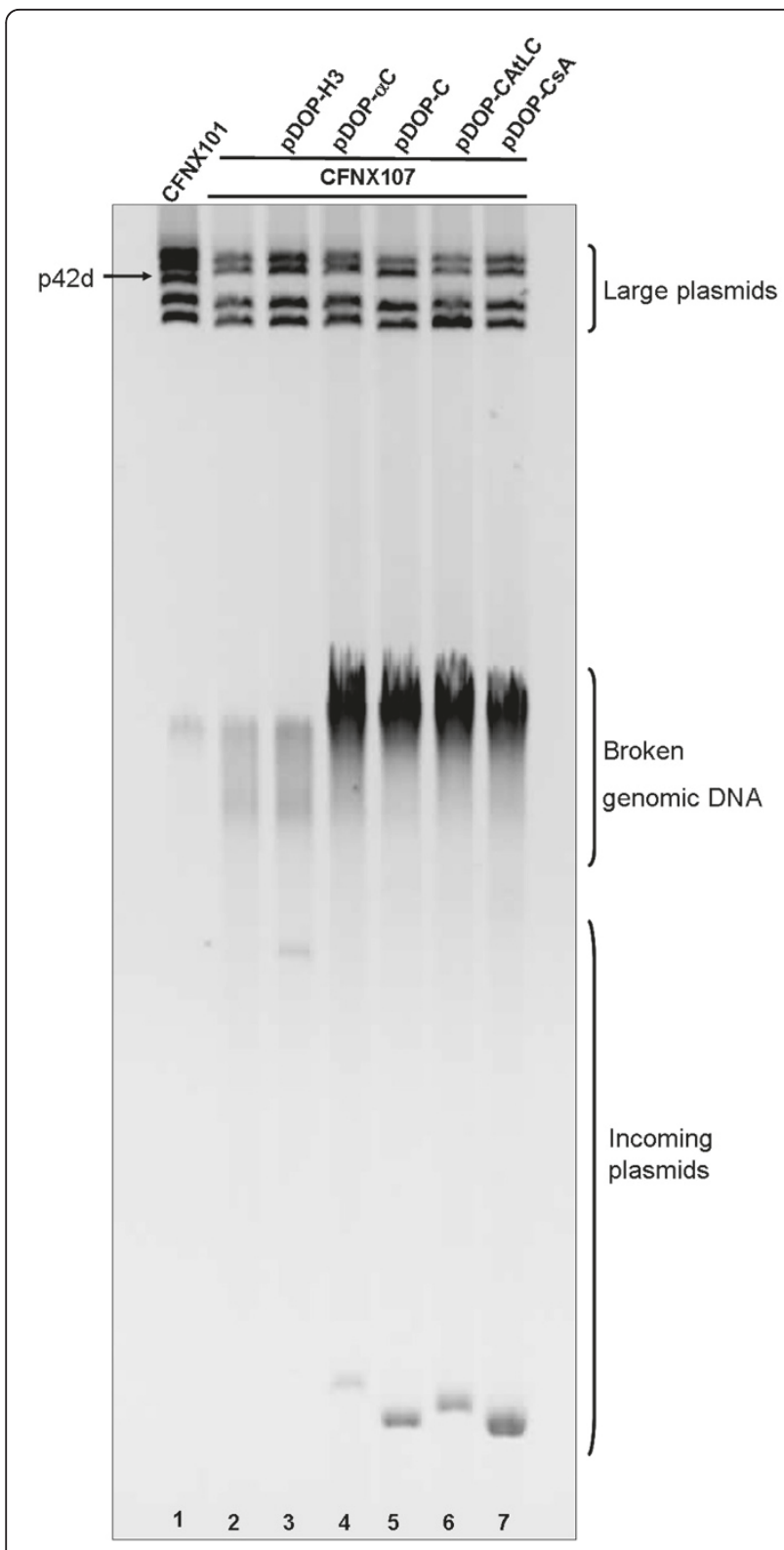

Figure 2 Plasmid profiles of Rhizobium etli CFNX101, and Rhizobium etli CFNX107 transconjugants, carrying the following plasmids: pDOP-H3, pDOP- $\alpha \mathrm{C}$, pDOP-C, pDOP-CAtLC, pDOP-CsA. Brackets at right show the positions of the resident large plasmids, broken DNA, and of the incoming plasmids. Arrow at left shows the location of plasmid p42d, in R. etli CFNX101. Negative image of Ethidium bromide stained gel.

search for three sequence features that are characteristic of the oriV in low copy-number plasmids: a set of tandem direct repeat sequences (iterons), a region of high $\mathrm{A}+\mathrm{T}$ content, and DnaA boxes. We only detected a region of high $\mathrm{A}+\mathrm{T}$ content between positions 450 and 850 of the repC coding region. However, we did not find any trace of even highly degenerated direct repeat sequences or of DnaA boxes.

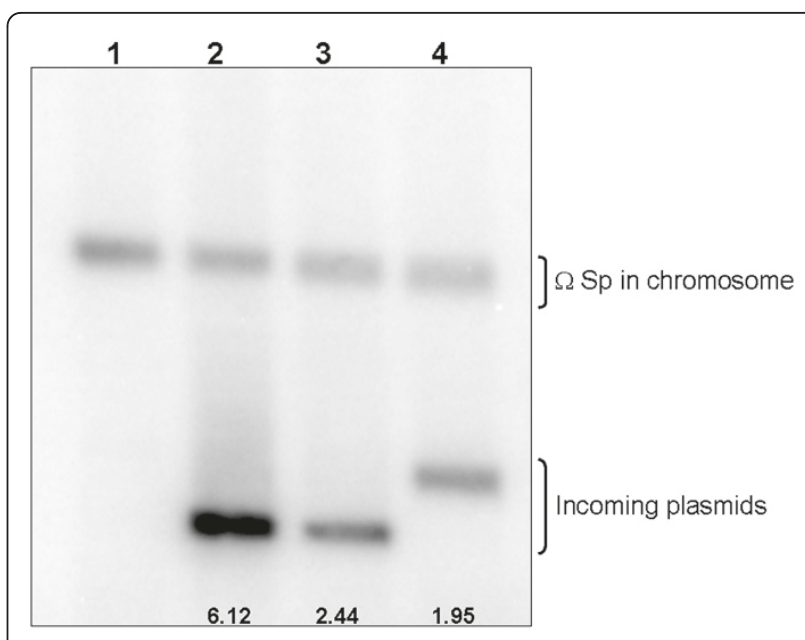

Figure 3 Plasmid copy number. Autoradiogram of a Southern blot of total DNA digested with HindIII and probed simultaneously with The $\Omega$-Spc cassette, located within recA gene (chromosomal detector) and with a pDOP vector (incoming plasmid detector). The plasmid copy number of each strain was calculated as the ratio of the integrated hybridization signal of repC (incoming plasmid) and the integrated hybridization signal of $\Omega$-Spc cassette (chromosome). Lane 1, CFNX107; lane 2, CFNX107/pDOP-C; lane 3, CFNX107/pDOP$\alpha \mathrm{C}$; lane 4, pDOP-H3. Numbers at the bottom indicate the plasmid/ chromosome ratio.

To determine if the high $\mathrm{A}+\mathrm{T}$ content region has a role in plasmid replication, we constructed a $r e p C$ derivative in which a group of silent mutations were introduced with the aim of altering the $\mathrm{A}+\mathrm{T}$ content and increase the DNA duplex stability of this region, without disrupting the $\operatorname{rep} C$ product (Figure 5). This repC mutant was cloned into pDOP under the Plac promoter and a SD sequence, generating the plasmid pDOPTtMC. This plasmid could not replicate in Rhizobium

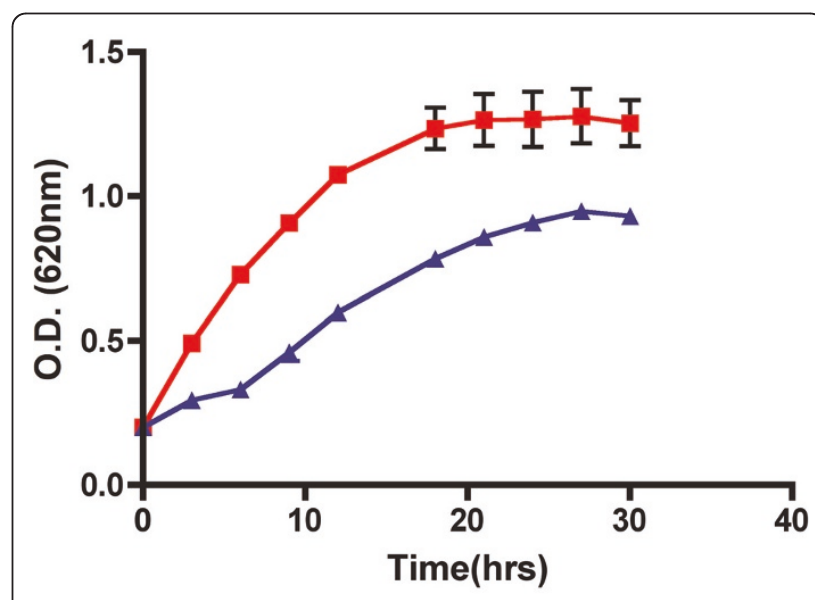

Figure 4 Growth kinetics of $R$. etli CFNX107 (red line), and R. etli CFNX107/pDOP-C (blue line), in PY medium without antibiotics, incubated at $30^{\circ} \mathrm{C}$, and $250 \mathrm{rpm}$ (see Methods). 
a)

$\int_{V}^{658} \mathrm{nt}$ repC GTT CTC AAT GAA ATG GAG ATG CTC CGT GAG GAA GTG CTT AAT CGT TTG TtMC GTG CTC AAC GAG ATG GAG ATG CTC CGC GAG GAG GTG CTG AAC CGC TTG

$\begin{array}{lllllllllllllllll}\mathrm{E} & \mathrm{N} & \mathrm{L} & \mathrm{E} & \mathrm{N} & \mathrm{A} & \mathrm{E} & \mathrm{K} & \mathrm{I} & \mathrm{S} & \mathrm{T} & \mathrm{H} & \mathrm{A} & \mathrm{A} & \mathrm{H} & \mathrm{I}\end{array}$ TEPC GAA AAC CTC GAG AAT GCT GAA AAA ATT AGC ACC AAT GCT GCT CAT ATC TtMC GAG AAC CTC GAG AAC GCC GAG AAG ATC AGC ACC AAC GCC GCC CAC ATC

$\begin{array}{llllllllllllllll}E & Q & H & I & Q & N & S & K & P & E & S & V & H & E & S & E\end{array}$ repC GAG CAG CAC ATA CAG AAT TCA AAA CCC GAA TCC GTA AAT GAA TCT GAג TtMC GAG CAG CAC ATC CAG AAC TCC AAG CCC GAG TCC GTG AAC GAG TCC GAG

$\begin{array}{lllllllllllll}P & R & S & E & K & E & Q\end{array}$ repC CCA CGC TCC GAA AAG GAG CAG TtMC CCG CGC TCC GAG AAG GAG CAG

b)

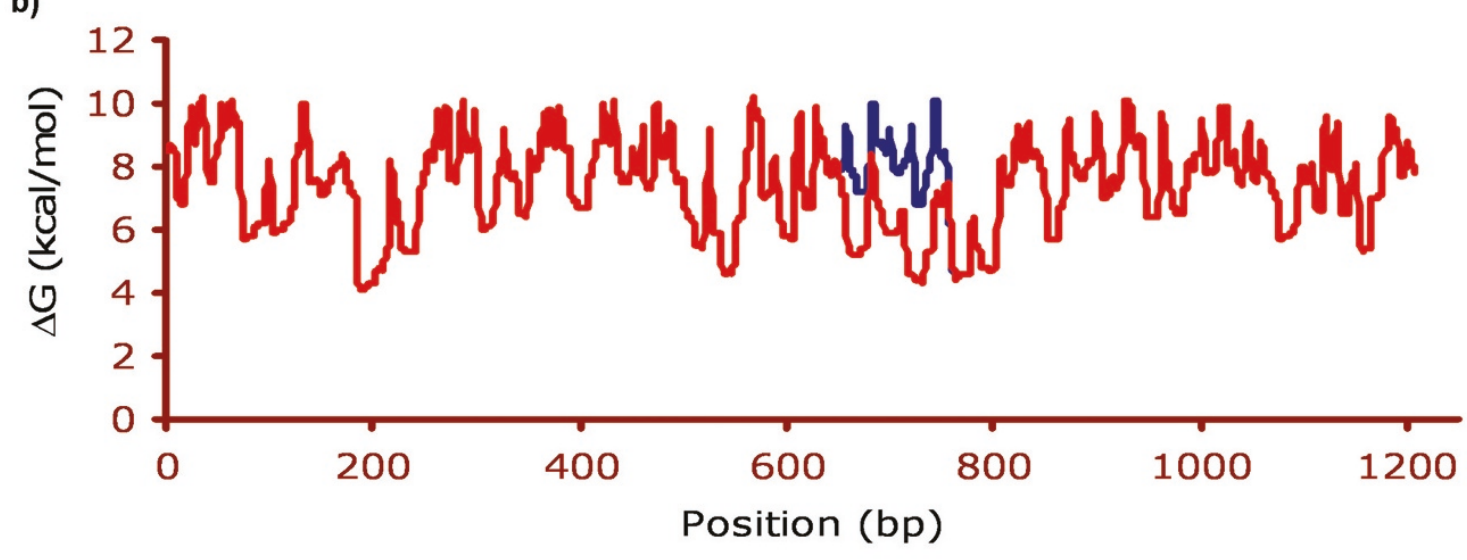

c) $r e p C$

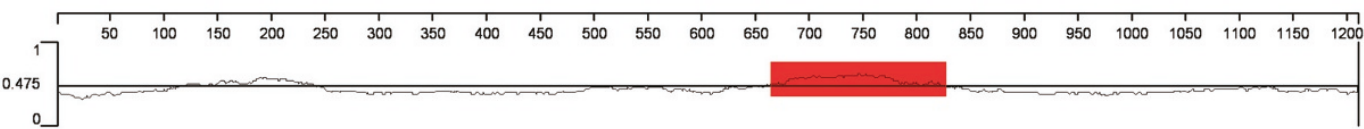

TtCH

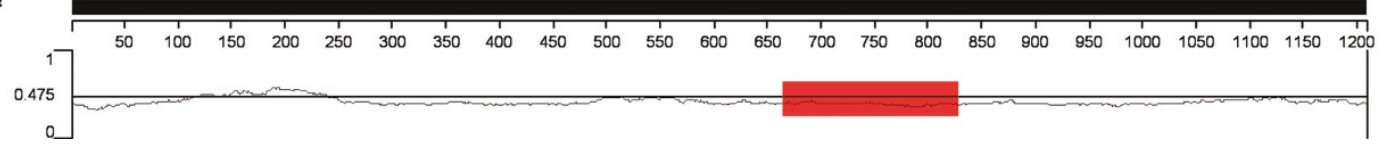

Figure 5 a) Gene alignment of repC and and its mutant derivative pDOP-TtMC from position 658 to 822 , indicating nucleotide changes introduced into pDOP- TtMC (red letters) to increase the $\mathrm{C}+\mathrm{G}$ content of this region. Note that the included mutations did not change the RepC protein sequence. b) DNA duplex stability expressed as $\triangle \mathrm{G}$ along repC gene (red line) and its mutant derivative TtMC (blue line). c) Graphic showing A+T content along repC gene and its mutant derivative TtMC. A+T average in both genes is the same: 0.475 . The A+T rich region of repC is boxed. Note that the equivalent region in TtMC, also boxed, the A+T content is above the average. 
strains with or without $\mathrm{p} 42 \mathrm{~d}$, indicating that the $\mathrm{A}+\mathrm{T}$ rich region plays a major role in replication.

\section{RepC exerts its action in cis}

The identification of an ori $V$ sequence is generally based on its ability to facilitate replication when present on a plasmid that otherwise could replicate only if the appropriate replication factors (e.g., an initiator protein) were provided in trans. To more precisely locate the oriV within $r e p C$, we cloned a collection of internal segments of repC into the suicide vector pDOP (Figure 1). This collection was conjugated into an $R$. etli strain containing the parental plasmid (CFNX101) as the source of all the trans elements required for replication, but we were unable to obtain transconjugants.

To determine if the activation of ori $V$ requires transcription (i.e., the repC mRNA also acts as a replication primer), we constructed a pDOP derivative that contained a repC gene but lacked a SD sequence (pDOP$\mathrm{Cs} / \mathrm{SD}$ ) (Figure 1). This plasmid was also incapable of replicating in $R$. etli CFNX101. Similarly, the two plasmids with repC frame-shift mutations, pDOP-CBglII and pDOP-CSphI, were also conjugated into $R$. etli CFNX101 without success. Overall, these results indicate that $\mathrm{RepC}$ exerts its action in cis.

\section{RepC as an incompatibility factor}

Plasmid incompatibility, or the inability of two replicons to coexist in the same cell line, results from the sharing of elements involved in plasmid replication, partitioning or control [30]. The repC open reading frame of $\mathrm{p} 42 \mathrm{~d}$, when cloned in a vector capable of replicating in $R$. etli, CFNX101, can coexist with p42d [8]. However, all of our attempts to introduce the construct pDOP-C into $R$. etli CFNX101 failed. In contrast, CFNX101 transconjugants carrying a similar construct (pDOP-CsA) that contained the repC gene pSymA of $S$. meliloti 2011 were easily obtained. The frequencies with which CFNX101/pDOPCsymA and CFNX107/pDOP-CsymA transconjugants were obtained were similar (average $5 \times 10^{-3}$ ). Moreover, the plasmid profiles of the transconjugants showed that pDOP-CsA replicated in these strains as an independent entity. These observations indicate that $\mathrm{pDOP}-\mathrm{C}$ and its parental plasmid p42d are incompatible, while that of pDOP-CSymA and $\mathrm{p} 42 \mathrm{~d}$ are compatible.

The RepC protein of S. meliloti 2011 pSymA shares $54 \%$ identity with the $\mathrm{p} 42 \mathrm{~d}$ RepC protein, and both proteins have very similar secondary structures (Figure 6). To map the RepC regions of $\mathrm{p} 42 \mathrm{~d}$ that are involved in plasmid incompatibility, a collection of hybrid genes containing fragments of the repC genes from S. meliloti pSymA and $R$. etli $\mathrm{p} 42 \mathrm{~d}$ were constructed. A schematic representation of the hybrid genes and their properties is shown in Figure 7. The hybrid genes were designed so that none of the predicted alpha-helix and beta regions of the $r e p C$ products were disturbed. The hybrid genes were cloned into pDOP under the Plac promoter and transferred by conjugation into $R$. etli CFNX107 to determine their ability to replicate autonomously and into $R$. etli CFNX101 to test if they were able to replicate without the interference of $\mathrm{p} 42 \mathrm{~d}$. Two constructs were capable of replicating in both genetic backgrounds: pDOP-C1-990 and pDOP-C1-1086. The rest of the constructs failed to replicate in both strains. The plasmid pDOP-C1-1086 expresses a hybrid protein containing the first 362 amino acid residues (aa) of the $\mathrm{p} 42 \mathrm{~d} \mathrm{RepC}$ protein and the last 39 aa carboxy-terminal region of the pSymA RepC protein. With respect to plasmid incompatibility, this recombinant plasmid behaved the same as plasmid pDOPCSymA, i.e., it replicated similarly in the strains CFNX101 and CFNX107. This result indicates that the $\mathrm{RepC}$ region involved in plasmid incompatibility resides in the last 39 amino acid residues of the protein.

\section{Discussion}

Plasmids in which the oriV is located in the gene encoding an initiation protein are uncommon but not exceptional. The Enterococcus faecalis pheromone-responding plasmid pAD1 [31] (Francia, et al., 2004), the Staphylococcus xylosus plasmid pSX267 [32], the plasmids pAM 31 and pLS32 from Bacillus subtilis [33-35], and the Staphylococcus aureus multiresistance plasmids pSK1 and pSK41 $[36,37]$ fall into this category. However, the origins of replication in all of these plasmids have recognizable iterons, and an insert that contains some or all of the iterons from these plasmids is usually capable of driving plasmid replication if the initiator protein is provided in trans. The minimal replicon of the $\mathrm{p} 42 \mathrm{~d}$ plasmid is the repC ORF sequence driven by a constitutive promoter (Plac) with an SD sequence that we designed. Frame shift and deletion mutants of the repC gene disrupted the capacity for replication of the minimal replicon, indicating that $\mathrm{RepC}$ is essential for replication and is likely the initiator protein. To confirm this function, it will be necessary to demonstrate that this protein binds the ori $V$, melts the doublestranded DNA, and recruits the initiation host factor.

A DNA sequence analysis of the repC gene clearly showed the absence of iterons or other large, perfect or imperfect, repetitive sequences ( $>8 \mathrm{bp}$ ), which are the typical DNA-binding sites of plasmid initiator proteins [1].

The replication of several bacterial plasmids, such as P1, F, R6K, RK2, Rts1, pMU720, and pSC101, requires a crucial and concerted participation of DnaA and the plasmid-encoded initiator protein. These plasmids contain at least one DnaA box in their ori $V$ sequences [38-43]. For other plasmids, DnaA participates only as an accessory, but these plasmids also contain DnaA boxes in their origins of replication (e.g., pR1) [44]. 
However, we failed to identify such DnaA boxes within the repC-coding region, suggesting that DnaA does not have a role in $\mathrm{p} 42 \mathrm{~d}$ replication.

A common property of theta-replicating plasmids is an $\mathrm{A}+\mathrm{T}$ rich region close to the origin of replication, which is necessary for strand melting and the assembly of host initiation factors [1]. The repC ORF sequence of $\mathrm{p} 42 \mathrm{~d}$ contains a large $\mathrm{A}+\mathrm{T}$ rich region that is crucial for plasmid replication. A construct carrying silent mutations that partially eliminated the $\mathrm{A}+\mathrm{T}$ rich region was 
a)

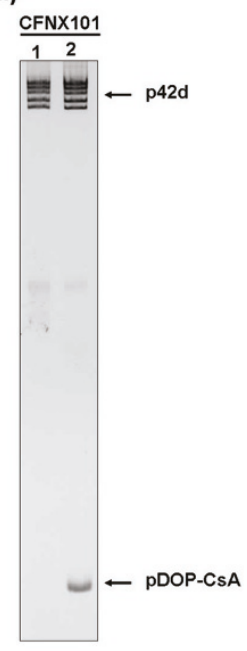

b)

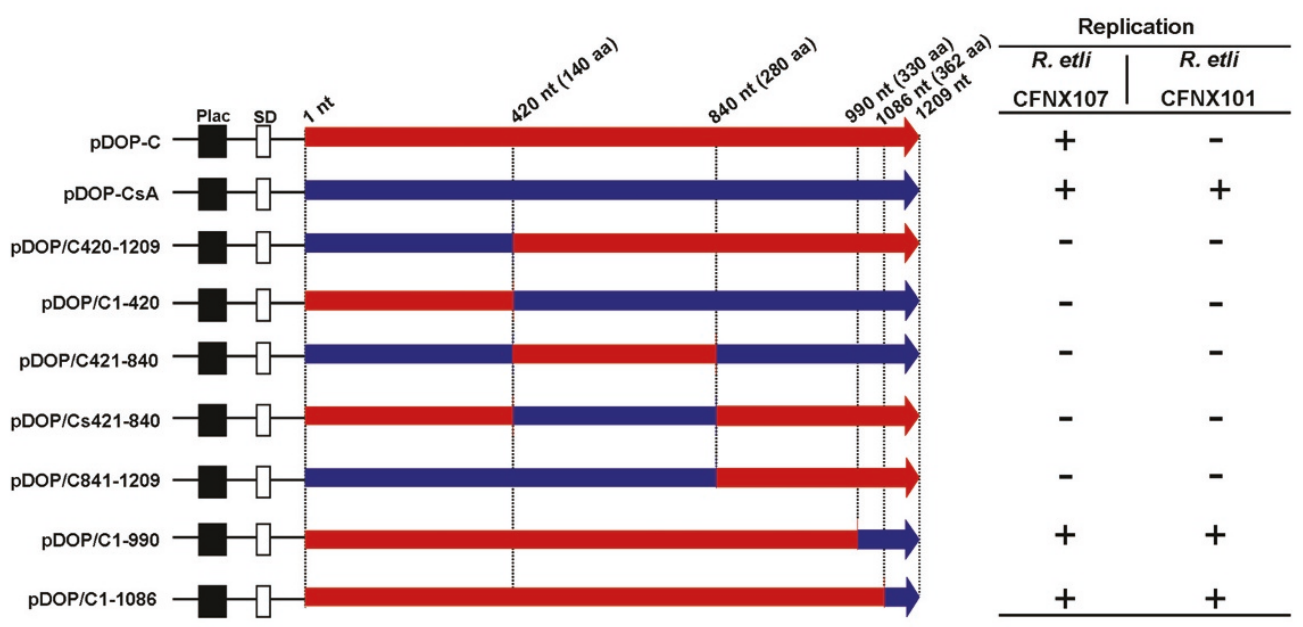

Figure 7 a) Plasmid profiles of CFNX101 (lane 1) and CFNX101/pDOP-CsA (lane 2), showing that plasmid p42d and pDOP-CSA are compatible. b) Linear representation of constructs containing SymA repC gene (blue arrow), p42d repC gene (red arrow) and SymA/p42d hybrid derivatives (blue/red arrows), and their associated replication capabilities when introduced into R. etli CFNX101 (with p42d) and CFNX107 (a p42d cured derivative) strains (table at left). " + " Symbols indicate that the construct are capable to replicate, and " - " that the construct is incapable to do that. Construct names are listed at the right of the figure. Black squares indicate the relative position of the Plac promoter, and the white rectangles the position of the Shine-Dalgarno (SD) sequences. Numbers at top indicate the positions where the SymA/p42d regions were swap.

unable to promote replication in $R$. etli strains with or without the symbiotic plasmid, indicating that this region is an essential part of the oriV. However, a sequence analysis of other repC genes located in rep $A B C$ operons revealed that an $\mathrm{A}+\mathrm{T}$ rich region was present in all of the analyzed plasmids but its relative location was not conserved (data not shown).

The $\mathrm{p} 42 \mathrm{~d}$ minimal replicon (pDOP-C) has two intriguing properties. First, the construct resulted in enhancing the plasmid copy-number to around six, in contrast parental plasmid, which was maintained at 1-2 copies per chromosome. Second, the strain carrying this construct has a longer duplication time and a lower yield when the cells reach stationary phase than the strain without this construct.

While describing the observed increase in the plasmid copy-number, we must bear in mind that the rep $C$ gene in $\mathrm{pDOP}-\mathrm{C}$ was expressed by a constitutive promoter.

In addition, the negative transcriptional regulation of the repC gene expression mediated by RepA and RepB was eliminated, and the antisense RNA (ctRNA), which also plays a negative role in the expression of repC, was removed. In the absence of these layers of negative regulation, it is expected that the plasmid replication would accelerate resulting in the production of new DNA molecules with a concomitant increase in the number of new origins of replication, which in turn, could be used to promote new rounds of replication, leading to cell death. However, in the present study, with the use of the minimal replicon (pDOP-C) we did not observe cell death, and the plasmid copy-number increased only moderately. This observation suggests the existence of a posttranslational mechanism that limits RepC activity, thus preventing over-initiation.

Growth kinetics of CFNX101 and CFNX107 were identical (data not shown), however, when pDOP-C was introduced into CFNX1017 growth of the bacterium was inhibited. The growth rate and yield diminution observed in strain CFNX107/pDOP-C relative to CFNX107 is not likely caused by the metabolic burden imposed by pDOP-C replication. The size of the parental plasmid (p42d) is approximately $374 \mathrm{~Kb}$, while the size of pDOP-C is approximately $5.57 \mathrm{~Kb}$; even if we take into consideration the 6-fold increase in plasmid copy-number, the amount of DNA required for replication in CFNX107/pDOP-C is several fold lower than the amount of DNA required for replication in CFNX101. Based on these observations it can be hypothesized that RepC, being an initiator protein, must perform three tasks: recognize the origin of replication, unwind the DNA at the origin, and recruit the replisome. An excess of RepC could lead to the formation of more of replication "bubbles". However, if one or more elements of the replisome are suboptimal in the growing cell, then, some replication forks will be stalled resulting in inhibition of cell division and growth.

We demonstrated that pDOP-C was capable of autonomous replication in an $R$. etli strain lacking the 
parental plasmid (p42d). However, we could not introduce this construct into an $R$. etli strain harboring the parental plasmid. In contrast, a similar construct that contained the repC gene of $S$. meliloti pSymA replicated autonomously with the same behavior in both strains. This result indicates that $\mathrm{RepC}$ is an incompatibility factor that prevents the coexistence of $\mathrm{p} 42 \mathrm{~d}$ and pDOP-C and that the incompatibility phenomenon is replicon-specific. Additionally, a construct (pDOP-C11086) expressing a chimeric protein consisting of the amino-terminal region of $\mathrm{p} 42 \mathrm{RepC}$ and 39 aa residues of the carboxy-terminal region of the pSymA RepC protein was capable of replicating as an independent entity with the same efficiency in $R$. etli strains, with or without $\mathrm{p} 42 \mathrm{~d}$. This result indicates that the last 39 aa residues of the $\mathrm{RepC}$ carboxy-terminal region are directly involved in the incompatibility phenotype. A close inspection of this region in the RepC proteins of pSymA and p42d shows that they share $62.5 \%$ of identity, indicating that 15 amino acid residues or less are critical in promoting the incompatibility phenotype. Interestingly, however, in spite of the variations in 15 aa residues, RepC proteins of $\mathrm{p} 42 \mathrm{~d}$ and $\mathrm{pSymA}$ have a similar secondary structure: both possess two alpha helices of ten amino acid residues each, separated by a coiled region of six amino acid residues, in the same relative positions.

Our current hypothesis linking incompatibility and the $\mathrm{RepC}$ posttranslational regulation is as follows: $\mathrm{RepC}$, like many other plasmid-encoded initiator proteins, exists in two forms, an active monomer and an inactive dimer, and protein thermodynamics favors dimer formation [1]. The RepC carboxy-terminal region is involved in dimer formation, and the dimerization process is replicon-specific. The introduction of pDOP-C into a strain containing $\mathrm{p} 42 \mathrm{~d}$ displaces the $\mathrm{RepC}$ monomerdimer equilibrium that favors the inactive form, preventing the establishment of the incoming plasmid. A similar introduction of a construct with the RepC of a compatible plasmid will not affect the monomer-dimer equilibrium and will allow the establishment of the new plasmid.

Another unusual observation was the inability to complement the repC ORF in trans for replication. One possibility is that the $\operatorname{rep} C$ transcript acts as an RNA primer for replication or assists in DNA melting at the oriV. However, the construct pDOP-Cs/SD, which lacks a SD sequence, could not replicate in CFNX101, suggesting that translation is required for the newly synthesized RepC protein to be located at the oriV. To the best of our knowledge, the only initiator protein that functions only in cis is RepA from prophage N15 [45]. At this stage we cannot determine which of these possibilities is more likely, and further experiments are needed to resolve these questions.

\section{Conclusions}

$\mathrm{RepC}$ is the only element encoded in the $\operatorname{rep} A B C$ operon of the Rhizobium etli p42d plasmid that is necessary and sufficient for plasmid replication and is likely the initiator protein. The oriV of this plasmid resides within the $r e p C$ gene and is located close to or inside of a large $\mathrm{A}+\mathrm{T}$ region. This architecture is shared by other rep $A B C$ plasmids. Our results also indicate that $\mathrm{RepC}$ can act as an incompatibility factor and that the last 39 aa of the carboxy-terminal region of this protein are involved in this phenotype.

\section{Acknowledgements and Funding}

This work was supported by the Consejo Nacional de Ciencia y Tecnología (CONACyT, México) (Grant number: 000000000100099$) ;$ and by the Programa de Apoyo a Proyectos de Investigación e Inovación Tecnológica (PAPIITUNAM, México) (Grant number IN205611-3) to M.A. C. R. C-R, F. P-L and G P$S$ were supported during the Ph.D. program (Programa de Doctorado en Ciencias Biomédicas-Universidad Nacional Autónoma de México) with scholarships from Consejo Nacional de Ciencia y Tecnología and Dirección General de Estudios de Posgrado (México). We are greatly indebted to Ángeles Pérez-Oseguera for her technical support, and to Dr. Pallavolu Maheswara Reddy for his critical review of the manuscript.

\section{Authors' contributions}

$R$ C-R conducted the bulk of the experiments and made the constructions; $F$ $P-L$ and G P-S made growth kinetics, plasmid profiles and incompatibility experiments. MAC designed and coordinated the study, and wrote the manuscript. All authors read and approved the final manuscript.

\section{Competing interests}

The authors declare that they have no competing interests.

Received: 15 April 2011 Accepted: 30 June 2011

Published: 30 June 2011

\section{References}

1. del Solar G, Giraldo R, Ruiz-Echevarría MJ, Espinosa M, Díaz-Orejas R: Replication and control of circular bacterial plasmids. Microbiol Mol Biol Rev 1998, 62:434-464.

2. Nordström K, Molin S, Light J: Control of replication of bacterial plasmids: genetics, molecular biology, and physiology of the plasmid R1 system. Plasmid 1984, 12:71-90.

3. Paulsson J, Chattoraj DK: Origin inactivation in bacterial DNA replication control. Mol Microbiol 2006, 61:9-15.

4. Zakrzewska-Czerwinska J, Jakimowicz D, Zawilak-Pawlik A, Messer W: Regulation of the initiation of chromosomal replication in bacteria. FEMS Microbiol Rev 2007, 31:378-387.

5. Cevallos MA, Cervantes-Rivera R, Gutiérrez-Ríos RM: The repABC plasmid family. Plasmid 2008, 60:19-37.

6. Castillo-Ramírez S, Vázquez-Castellanos JF, González V, Cevallos MA: Horizontal gene transfer and diverse functional constrains within a common replication-partitioning system in Alphaproteobacteria: the repABC operon. BMC Genomics 2009, 10:536.

7. Pappas KM: Cell-cell signaling and the Agrobacterium tumefaciens $\mathrm{Ti}$ plasmid copy number fluctuations. Plasmid 2008, 60:89-107.

8. Ramírez-Romero MA, Soberón N, Pérez-Oseguera A, Téllez-Sosa J, Cevallos MA: Structural elements required for replication and incompatibility of the Rhizobium etli symbiotic plasmid. J Bacterio/ 2000, 182:3117-3124. 
9. Pappas KM, Winans SC: The RepA and RepB autorepressors and TraR play opposing roles in the regulation of a Ti plasmid repABC operon. $\mathrm{Mol}$ Microbiol 2003, 49:441-455.

10. Gerdes K, Moller-Jensen J, Jensen RB: Plasmid and chromosome partition: surprises from phylogeny. Mol Microbiol 2000, 37:455-466.

11. Ramírez-Romero MA, Téllez-Sosa J, Barrios H, Pérez-Oseguera A, Rosas V, Cevallos MA: RepA negatively autoregulates the transcription of the repABC operon of the Rhizobium etli symbiotic plasmid basic replicon. Mol Microbiol 2001, 42:195-204.

12. Tabata S, Hooykaas PJ, Oka A: Sequence determination and characterization of the replicator region in the tumor-inducing plasmid pTiB6S3. J Bacteriol 1989, 171:1665-1672.

13. Bartosik D, Baj J, Wlodarczyk M: Molecular and functional analysis of pTAV320, a repABC-type replicon of the Paracoccus versutus composite plasmid pTAV1. Microbiology 1998, 144:3149-3157.

14. Bartosik D, Szymanik M, Wysocka E: Identification of the partitioning site within the repABC-type replicon of the composite Paracoccus versutus plasmid pTAV1. J Bacteriol 2001, 183:6234-6243.

15. Soberón N, Venkova-Canova T, Ramírez-Romero MA, Téllez-Sosa J, Cevallos MA: Incompatibility and the partitioning site of the repABC basic replicon of the symbiotic plasmid from Rhizobium etli. Plasmid 2004, 51:203-216.

16. Chai Y, Winans SC: RepB protein of an Agrobacterium tumefaciens Ti plasmid binds to two adjacent sites between repA and repB for plasmid partitioning and autorepression. Mol Microbiol 2005, 58:1114-1129.

17. MacLellan SR, Zaheer R, Sartor AL, MacLean AM, Finan TM: Identification of a megaplasmid centromere reveals genetic structural diversity within the repABC family of basic replicons. Mol Microbiol 2006, 59:1559-1575.

18. Chai $Y$, Winans SC: A small antisense RNA downregulates expression of an essential replicase protein of an Agrobacterium tumefaciens $\mathrm{Ti}$ plasmid. Mol Microbiol 2005, 56:1574-1585.

19. MacLellan SR, Smallbone LA, Sibley CD, Finan TM: The expression of a novel antisense gene mediates incompatibility within the large repABC family of alpha-proteobacterial plasmids. Mol Microbiol 2005, 55:611-623.

20. Venkova-Canova T, Soberón NE, Ramírez-Romero MA, Cevallos MA: Two discrete elements are required for the replication of a rep $A B C$ plasmid: an antisense RNA and a stem-loop structure. Mol Microbiol 2004, 54:1431-1444.

21. Cervantes-Rivera R, Romero-López C, Berzal-Herranz A, Cevallos MA: Analysis of the mechanism of action of the antisense RNA that controls the replication of the repABC plasmid p42d. I Bacteriol 2010, 192:3268-3278.

22. Noel KD, Sanchez A, Fernandez L, Leemans J, Cevallos MA: Rhizobium phaseoli symbiotic mutants with transposon Tn5 insertions. J Bacteriol 1984, 158:148-155

23. Simon R, Priefer U, Pühler A: A broad host-range mobilization system for in vivo genetic engineering transposon mutagenesis in Gram negative bacteria. Bio/Technology 1983, 1:784-791.

24. Ramírez-Romero MA, Bustos P, Girard L, Rodríguez O, Cevallos MA, Dávila G Sequence, localization and characteristics of the replicator region of the symbiotic plasmid of Rhizobium etli. Microbiology 1997, 143:2825-2831.

25. Horton RM, Hunt HD, Ho SN, Pullen JK, Pease LR: Engineering hybrid genes without the use of restriction enzymes: gene splicing by overlap extension. Gene 1989, 77:61-68.

26. Hynes MF, McGregor NF: Two plasmids other than the nodulation plasmid are necessary for formation of nitrogen-fixing nodules by Rhizobium leguminosarum. Mol Microbiol 1990, 4:567-574.

27. Thompson JD, Higgins DG, Gibson TJ: CLUSTAL W: improving the sensitivity of progressive multiple sequence alignment through sequence weighting, position-specific gap penalties and weight matrix choice. Nucleic Acids Res 1994, 22:4673-4680.

28. Jones DT: Protein secondary structure prediction based on positionspecific scoring matrices. J Mol Biol 1999, 292:195-202.

29. Huang Y, Kowalski D: WEB-THERMODYN: sequence analysis software for profiling DNA helical stability. Nucl Acids Res 2003, 31:3819-3821.

30. Novick RP: Plasmid incompatibility. Microbiol Rev 1987, 51:381-395.

31. Francia MV, Fujimoto S, Tille P, Weaver KE, Clewell DB: Replication of Enterococcus faecalis pheromone-responding plasmid pAD1: location of the minimal replicon and oriV site and RepA involvement in initiation of replication. J Bacteriol 2004, 186:5003-5016.

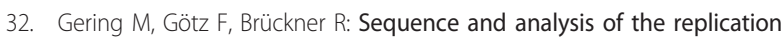
region of the Staphylococcus xylosus plasmid pSX267. Gene 1996, 182:117-122

33. Bruand C, Ehrlich SD: Transcription-driven DNA replication of plasmid pAMbeta1 in Bacillus subtilis. Mol Microbiol 1998, 30:135-145.

34. Tanaka T, Ogura M: A novel Bacillus natto plasmid pLS32 capable of replication in Bacillus subtilis. FEBS Lett 1998, 422:243-246.

35. Tanaka T, Ishida H, Maehara T: Characterization of the replication region of plasmid pLS32 from the Natto strain of Bacillus subtilis. J Bacteriol 2005, 187:4315-4326

36. Kwong SM, Skurray RA, Firth N: Staphylococcus aureus multiresistance plasmid pSK41: analysis of the replication region, initiator protein binding and antisense RNA regulation. Mol Microbiol 2004, 51:497-509.

37. Kwong SM, Skurray RA, Firth N: Replication control of staphylococcal multiresistance plasmid pSK41: an antisense RNA mediates dual-level regulation of Rep expression. J Bacteriol 2006, 188:4404-4412.

38. Betteridge T, Yang J, Pittard AJ, Praszkier J: Role of RepA and DnaA proteins in the opening of the origin of DNA replication of an IncB plasmid. J Bacteriol 2004, 186:3785-3793.

39. Gaylo PJ, Turjman N, Bastia D: DnaA protein is required for replication of the minimal replicon of the broad-host-range plasmid RK2 in Escherichia coli. J Bacteriol 1987, 169:4703-4709.

40. Hansen EB, Yarmolinsky MB: Host participation in plasmid maintenance: dependence upon $\operatorname{dnaA}$ of replicons derived from P1 and F. Proc Natl Acad Sci USA 1986, 83:4423-4427.

41. Hasunuma K, Sekiguchi M: Replication of plasmid pSC101 in Escherichia coli K12: requirement for dnaA function. Mol Gen Genet 1977, 154:225-230

42. Itoh Y, Terawaki Y: Replication properties of mini-Rts1 derivatives deleted for DnaA boxes in the replication origin. Plasmid 1989, 21:242-246.

43. Kline BC, Kogoma T, Tam JE, Shields MS: Requirement of the Escherichia coli dnaA gene product for plasmid F maintenance. J Bacteriol 1986, 168:440-443.

44. Ortega-Jiménez S, Giraldo-Suárez R, Fernández-Tresguerres ME, BerzalHerranz A, Díaz-Orejas R: DnaA dependent replication of plasmid R1 occurs in the presence of point mutations that disrupt the $d n a A$ box of oriR. Nucleic Acids Res 1992, 20:2547-2551.

45. Mardanov AV, Ravin NV: Functional characterization of the repA replication gene of linear plasmid prophage N15. Res Microbiol 2006, 157:176-183.

46. Martínez-Salazar J, Romero D, Girard ML, Dávila G: Molecular cloning and characterization of the recA gene of Rhizobium phaseoli and construction of recA mutants. J Bacterio/ 1991, 173:3035-3040.

doi:10.1186/1471-2180-11-158

Cite this article as: Cervantes-Rivera et al:: The replication origin of a repABC plasmid. BMC Microbiology 2011 11:158.

\section{Submit your next manuscript to BioMed Central and take full advantage of:}

- Convenient online submission

- Thorough peer review

- No space constraints or color figure charges

- Immediate publication on acceptance

- Inclusion in PubMed, CAS, Scopus and Google Scholar

- Research which is freely available for redistribution

Submit your manuscript at www.biomedcentral.com/submit
C Biomed Central 